\title{
Treatment of bipolar disorder: a complex treatment for a multi-facet disorder
}

\author{
Konstantinos Fountoulakis*1, Eduard Vieta ${ }^{2}$, Melina Siamouli ${ }^{1}$, \\ Marc Valenti ${ }^{2}$, Stamatia Magiria ${ }^{1}$, Timucin Oral ${ }^{3}$, David Fresno ${ }^{2}$, \\ Panteleimon Giannakopoulos ${ }^{4}$ and George Kaprinis ${ }^{1}$
}

\begin{abstract}
Address: ${ }^{13 r d}$ Department of Psychiatry, Aristotle University of Thessaloniki, Greece, ${ }^{2}$ Bipolar Disorders Program, Hospital Clinic, University of Barcelona, IDIBAPS, Barcelona, Spain, 35th Inpatient Department of Psychiatry and Outpatient Unit of Mood Disorders, Bakirk $\phi y$ State Teaching and Research Hospital for Neuropsychiatry, Istanbul, Turkey and ${ }^{4}$ Department of Psychiatry, University of Geneva, Switzerland

* Corresponding author
\end{abstract}

from International Society on Brain and Behaviour: 3rd International Congress on Brain and Behaviour

Thessaloniki, Greece. 28 November - 2 December 2007

Published: 17 April 2008

Annals of General Psychiatry 2008, 7(SuppI I):S316 doi:I0.II86/I744-859X-7-SI-S316

This abstract is available from: http://www.annals-general-psychiatry.com/content/7/SI/S316

(c) 2008 Fountoulakis et al.; licensee BioMed Central Ltd.

Manic-depression or bipolar disorder is a multi-facet illness with an inevitably complex treatment. The current article summarizes the current status of our knowledge and practice in its treatment. It is widely accepted that lithium is moderately useful during all phases of bipolar illness and it might possess a specific effectiveness on suicidal prevention. Both first and second generation antipsychotics are widely used and the FDA has approved olanzapine, risperidone, quetiapine, ziprasidone and aripiprazole for the treatment of acute mania. They could also be useful in the treatment of bipolar depression however only limited data exist so far to support the use of quetiapine monotherapy or the olanzapine-fluoxetine combination. Some but not all anticonvulsants possess a broad spectrum of effectiveness, including mixed dysphoric and rapid-cycling forms. Lamotrigine may be effective in the treatment of depression but not mania. Antidepressant use is controversial. Guidelines suggest their cautious use in combination with an antimanic agent, because they are supposed to induce switching to mania or hypomania, mixed episodes and rapid cycling. The first-line psychosocial intervention in $\mathrm{BD}$ is psychoeducation, followed by cognitive-behavioral therapy. Other treatment options include Electroconvulsive therapy and transcranial magnetic stimulation. There is a gap between evidence base, which comes mostly from monotherapy trials, and clinical practice, where complex treatment regimens are the rule. 